\title{
ARTICLE
}

\section{Improving the Mesomorphic Behaviour of Supramolecular Liquid Crystals by Resonance-assisted Hydrogen Bonding}

Received 00th January 20xx, Accepted 00th January 20xx DOI: $10.1039 / x 0 x x 00000 x$

\author{
Marco Saccone, ${ }^{\text {ta }}$ Michael Pfletscher, ${ }^{\text {ta }}$ Sven Kather, ${ }^{a}$ Christoph Wölper, ${ }^{b}$ Constantin Daniliuc, ${ }^{c}$ \\ Markus Mezger, ${ }^{d}$ and Michael Giese ${ }^{a *}$
}

\begin{abstract}
A systematic structure-property relationship study on hydrogen-bonded liquid crystals was performed, revealing the impact of resonance-assisted hydrogen bonds (RAHBs) on the self-assembling behavior of the supramolecular architecture. The creation of a six-membered intramolecular hydrogen-bonded ring acts as a counterpart to the selforganization between hydrogen bond donators and acceptors and determines thus the suprastructure. Variation of the hydrogen-bonding pattern allowed us to significantly improve the temperature range of the reported liquid crystalline assemblies.
\end{abstract}

\section{Introduction}

Within the past decades supramolecular chemistry has evolved into a powerful tool for the design of new functional materials. ${ }^{1}$ The supramolecular approach provides a facile access to a plethora of new materials, since here, suitable molecules are held together by specific non-covalent interactions and may introduce novel properties caused by the self-assembled structure. In addition, the dynamics of the noncovalent interactions between the building blocks provides the ability for reversible response to changes in the environment or self-repair behaviour. ${ }^{2}$ Especially hydrogen bonds, which play a key role in molecular processes in nature, due to their dynamics, directionality, and tunability, ${ }^{3}$ are highly promising for the design of supramolecular liquid crystals. ${ }^{4}$

In this respect, Kato and Fréchet investigated self-assembled liquid crystals based on benzoic acid groups and pyridyl derivatives. ${ }^{5}$ Later, Bruce and collaborators demonstrated that also phenols are suitable hydrogen bond donors for the formation of supramolecular assemblies with liquid-crystalline properties. ${ }^{6}$ In the late 1980s, Percec et al. started a comprehensive study of hydrogen-bonded liquid crystalline materials. They reported on a hierarchical self-assembling of hydrogen bonded dendrimers and dendrons in complex suprastructures and deduced design principles for precise

\footnotetext{
Institute of Organic Chemistry and Cenide, University of Duisburg Essen,

Universitätsstraße 7, 45141 Essen, Germany.E-mail: michael.giese@uni-due.de

b. Institute of Inorganic Chemistry, University of Duisburg Essen, Universitätsstraße

7, 45141 Essen, Germany.

Institute of Organic Chemistry, Westfälische Wilhelms-Universität Münster

Corrensstrasse 40, 48149 Münster, Germany.

d. Institute of Physics, Johannes Gutenberg-Universität Mainz and Max-Planck-

Institut for Polymer Research, Ackermannweg 10, 55021 Mainz, Germany.

† These authors contributed equally to this work.

Electronic Supplementary Information (ESI) available: See
}

DOI: $10.1039 / \times 0 \times x 00000 x$ prediction of the 3D architecture. $^{7}$

Recently, we introduced a modular approach for comprehensive understanding of supramolecular liquid crystals with tailor-made properties. The reported semiflexible Hekates $^{8}$ are based on the self-assembly of phloroglucinol (PHG) with alkoxy-functionalized azopyridines (Ap) to yield hydrogen bonded liquid crystals. ${ }^{9}$ This allows us to gain considerable insight into the structure-property relationships of supramolecular liquid crystals en route to functional materials. ${ }^{10}$ A major limitation of the previously reported systems is the narrow temperature range of the liquid crystalline phase and the high transition temperature, which excluded this system from applications in functional materials. Therefore, we started systematic studies to investigate the impact of the substitution pattern of the hydrogen bond donating core, ${ }^{9 c}$ the linking group in the hydrogen bond accepting side chain ${ }^{9 \mathrm{~d}}$ and the fluorination degree and pattern on the liquid crystalline behaviour of the assemblies. $^{9 \mathrm{~b}}$

In the present study we investigate the impact of the functionalization of the hydrogen bond donating core on the liquid crystalline behaviour of the supramolecular Hekates. We therefore modified systematically the core unit ( $\boldsymbol{Y}$-PHG) and subsequently assembled with three azopyridines with different linear alkyl chains (Ap-n) to yield a series of hydrogen-bonded liquid crystals $\left(\boldsymbol{Y}\right.$-PHG $\cdots(\mathbf{A p}-\mathbf{n})_{3}$, Scheme 1$)$. This systematic approach identified resonance assisted hydrogen bonds $(\mathrm{RAHB})^{11}$ as promising tool for the stabilization of supramolecular liquid crystals, which was subsequently used to extend the temperature range up to $\Delta \mathrm{T}=50^{\circ} \mathrm{C}$ and lowered the $\mathrm{N} \rightarrow \mathrm{Cr}$ transition temperature below room temperature. 


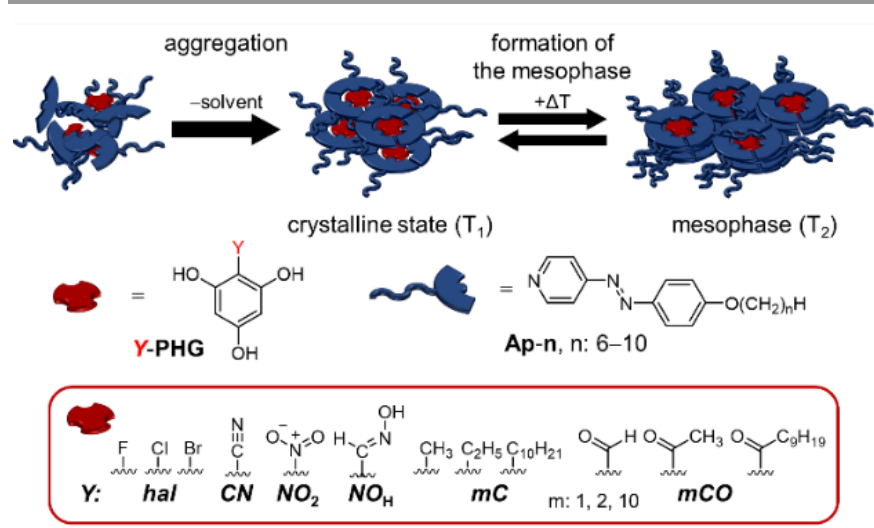

Scheme 1. The rationale of our approach highlight the self-assembly between Ap-n and Y-PHG cores. Cores were tuned to probe different effect as discussed in the text.

The 48 hydrogen-bonded assemblies were obtained (Scheme 1) and characterized by polarized optical microscopy (POM), differential scanning calorimetry (DSC) and small angle X-ray scattering (SAXS). The PHG $\cdots(\mathbf{A p}-\mathbf{n})_{3}$ assemblies serve as reference system. ${ }^{10 a}$ Full details about the synthesis and characterizations are given in the ESI. The groups introduced at the core unit were chosen in order to investigate different subtle effects, which might influence the liquid crystalline behaviour of the assemblies: the role of the dipole moment, molecular volume and steric hindrance at the hydrogen bond site. Dipole moments and volumes were obtained by density functional theory. ${ }^{12}$ As a definition of molecular volume we used the electronic charge encompassed by the $0.001 \mathrm{au}$ contour of the electron density. ${ }^{13}$ This choice is particularly relevant in the context of non-covalent interactions. ${ }^{14}$

\section{Discussion}

\section{Liquid crystalline behaviour of the assemblies}

Initially, the obtained hydrogen bonded assemblies were investigated by POM. The majority of the investigated assemblies homogeneously melted (except the 1:3 assemblies based on $\mathbf{N O}_{2}$-PHG and the mCO-PHG). Furthermore, most samples showed the characteristic Schlieren texture (Fig. $1 \mathrm{~A}$, C, D) of a nematic phase. The assemblies based on $\mathbf{m C O}$-PHG displayed fan-shaped textures as typical for smectic phases (Fig. 1 C), while the assemblies with CN-PHG showed both Schlieren and fan-shaped textures. The assignment of the mesophases by POM is supported by SAXS measurements.
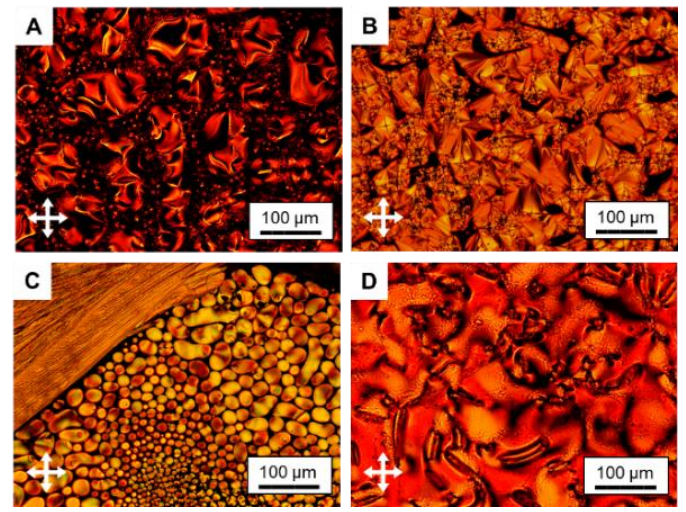

Fig. 1. Representative POM images of the HBAs under crossed polarizers showing the typical Schlieren $(A, D)$ and fan-shaped texture $(B, C)$ indicating nematic and smectic phases, respectively (A: Cl-PHG $\cdots(\text { Ap-9) })_{3}$. B: 1CO-PHG $\cdots(\text { Ap-9) })_{2}$. C: $\mathbf{N O}_{2}$-PHG $\cdots(\text { Ap-9 })_{1}$. D: NO ${ }_{\mathrm{H}}$-PHG $\left.\cdots(\mathrm{Ap}-9)_{3}\right)$.

However, the results obtained by POM were quantified by DSC (see Table S1-S15). In general, an increased range of the mesophases was observed in most of the substituted $\boldsymbol{Y}$-PHG assemblies compared to the non-substituted PHG series, underlining the importance of the substitution at the core moiety. However, the most interesting findings were found for PHG systems which are capable to form intramolecular hydrogen bonds. We found temperature ranges $(\Delta T)$ of more than $50{ }^{\circ} \mathrm{C}$ for the mesophases, which nearly double the mesophase range compared to the PHG assemblies. Figure 2 summarizes the results as obtained by POM and shows the temperature ranges of the mesophases for the 1:3 assemblies (Y-PHG:Ap-n; $n=6-10$ ) in dependence of the substituent at the hydrogen bond donating core moiety and chain length of the alkyl group.

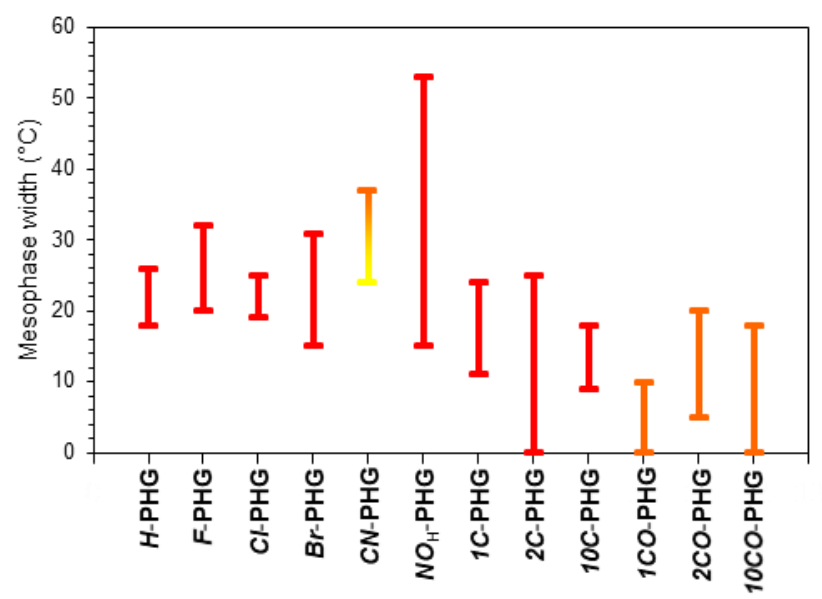

Fig. 2. Overview of the mesophase width using different $\boldsymbol{Y}$-PHG and Ap-n, n: 6, 8-10, reveals the dependency of liquid crystalline behaviour on the core functionalization and length of the terminal alkyl group. Yellow orange bar: smectic phases, red bar: nematic phase.

The focus of the present study is to improve the temperature range of the mesophase and to lower the transition temperatures. Since the most interesting findings were made for core moieties capable of forming intramolecular hydrogen bonds ( $\boldsymbol{Y}$-PHG, $\mathrm{Y}=\mathrm{NO}_{2}, \mathrm{NO}_{\mathrm{H}}, \mathrm{mCO}$ ) the following discussion 
will briefly summarize the results for all substituents and then focus on the systems which showed significant improvement of the mesomorphic behaviour due to the formation of RAHB.

Effect halogen atoms or cyano group: A common approach for conventional liquid crystals is to introduce a polar group to the molecular structure, which increases the electronic anisotropy and thereby stabilizes the liquid crystalline state. ${ }^{15}$ Halogens are known to have a tremendous effect on the properties of functional materials ${ }^{16}$ or the pharmaceutical effectiveness of drugs. ${ }^{17}$ The F-PHG assemblies reported earlier showed an increase nematic phase range compared to the PHG assemblies. Fluorine substitution has been indeed an effective tool to tune the properties of liquid crystalline assemblies, because it enhances the dipole moment of the molecule while barely modifying the molecular volume. ${ }^{\text {9e } 18,19}$

Also the Br-PHG assemblies showed enhanced mesophase range compared to the PHG assemblies. The CI-PHG assemblies, however, showed narrower temperature ranges for the nematic phase. Within the series of $\mathbf{C l}$-PHG assemblies, the temperature range of the nematic phase decreases with increasing alkyl chain length at the azopyridine. We attribute this behaviour to the features of the inductive and resonance effects in chlorine- and bromine-substituted benzenes (Fig. $2 \mathrm{~B}){ }^{20}$ which have been reported for covalent mesogens. ${ }^{21}$ Introducing a cyano group to the PHG unit yielded broader temperature ranges for the nematic phase $\left(\Delta T_{\max }=37^{\circ} \mathrm{C}\right)$ which are comparable to the results found for F-PHG or $\mathbf{B r}$ PHG. Attempts to correlate the dipole moment or the van der Waals volumes of the substituents with the mesomorphic behaviour of the assemblies did not reveal any systematic trend.

Effect of alkyl and alkanoyl chains: Since we are interested in lowering the transition temperatures of the hydrogen-bonded assemblies, making these materials appealing for potential applications, alkyl chains of different lengths (1C, 2C, 10C) were attached to the PHG core and the mesomorphic behaviour of its assemblies were studied. The introduction of a carbonyl group (1CO, 2CO, 10CO) should additionally enhance the ability of the assemblies to form smectic phases (Scheme 1). ${ }^{5 b}$

As expected the melting point of the core unit $\left(\mathrm{mp}_{\mathrm{PHG}}=215\right.$ ${ }^{\circ} \mathrm{C}$ ) was significantly lowered to 110 and $130^{\circ} \mathrm{C}$ for $10 \mathrm{C}$-PHG and 10CO-PHG, respectively. For the $\mathbf{m C}$-PHG-based assemblies, however, a systematic decrease in the mesophase range was observed upon increasing the alkyl chain length at the PHG core (Fig. 2D and 4). We attribute this to the increasing steric demand of the alkyl chains and a subsequent interference with the formation of the hydrogen-bonded assembly. The $\boldsymbol{m} \mathbf{C O}$-PHG assemblies revealed smectic $A$ phases with a temperature range of up to $\Delta \mathrm{T}_{S m A, \max }=20^{\circ} \mathrm{C}$ proven by POM and SAXS (Fig. 5, Supporting Fig. S58 and S59).

Effect of RAHB: Within the series of investigated assemblies we observed some unexpected behaviour. For example, systems based on the alkanoyl-substituted PHG core show the expected lowering of the transition temperature, widening the temperature range of the mesophase, however, was not observed. In addition, the $\mathbf{N O}_{2}$-PHG-based assemblies did not show any liquid crystalline behaviour (Fig. 2A and B), which is in contrast to findings of hydrogen-bonded mesogens reported by Bruce et al. ${ }^{22}$ In order to understand these discrepancies we started a comprehensive crystallographic study and cocrystallized a series of hydrogen-bonded assemblies, which provided insight into the supramolecular structure of the mesogens.

\section{Crystallography analysis}

The properties of the supramolecular assemblies are closely related to their hierarchical structure. ${ }^{1}$ Therefore, an insight into the structural features of the assemblies in the solid state is crucial for a comprehensive understanding of the properties of supramolecular materials. It should be noted that crystal structures are the result of various attractive and repulsive forces as well as packing effects, and we are well aware that the supramolecular arrangement of our systems may differ in the liquid crystalline phase. However, crystal structures provide a first idea of the architecture of the supramolecular assembly.

We were able to obtain single crystals for the 2CO-PHG core with Ap-3. Each asymmetric unit contains two assemblies, which surprisingly revealed the formation in the 1:2 ratio of core and side chain units. The distances of the $\mathrm{OH} \cdots \mathrm{N}_{\text {pyr }}$ hydrogen bonds correspond to $2.712-2.747 \AA$. The two azopyridine units of one assembly are aligned in a parallel fashion and show $\pi-\pi$ interactions (Fig 3B, red box). Further aromatic interactions come from two neighbouring cores and the anti-parallel aligned azopyridines in a tilted $T$-shaped variant (Fig. 3B, purple box). The third phenolic $\mathrm{OH}$-group of the core moiety forms an intramolecular hydrogen bond to the carbonyl group $\left(\mathrm{OH} \cdots \mathrm{O}_{\mathrm{CO}}=2.491-2.493 \AA\right.$ ) , preventing the 1:3 formation of the assembly with an additional azopyridine unit (Fig. 3A).

A

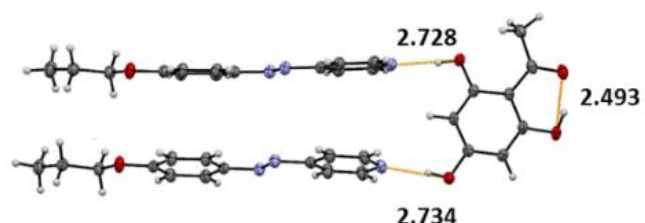

B

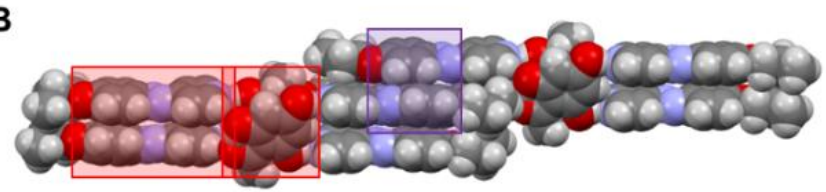

Fig. 3. Representative views of the molecular structures (A) and crystalline packing (B) of 2 CO-PHG $\cdots(\mathbf{A p}-\mathbf{3})_{2}$ showing $\pi-\pi$ interactions (red boxes) and a $T$-shaped alignment (purple box) of the side chain units. Ellipsoids are displayed at $50 \%$ probability. Distances are given in $\AA$. Only one of the two symmetrically independent units is displayed. 
Similar to the solid state structure of the alkanoyl-substituted PHG-derivatives the X-ray structure of the $\mathbf{N O}_{2}$-PHG assembly was obtained in a 1:1 ratio with Ap-8. The structure shows one hydrogen bond between the core and side chain $\left(\mathrm{OH} \cdots \mathrm{N}_{\text {pyr }}=2.667 \AA\right)$ and two resonance-assisted hydrogen bonds $\left(\mathrm{OH} \cdots \mathrm{O}_{\mathrm{NO} 2}=2.561,2.551 \AA\right.$; Fig. $\left.6 \mathrm{~A}\right)$ to the nitro group. The packing is favoured by other hydrogen bonds between neighbouring $\mathbf{N O}_{2}$-PHG cores via $\pi-\pi$ interactions (Fig. $6 \mathrm{~B}$ ).

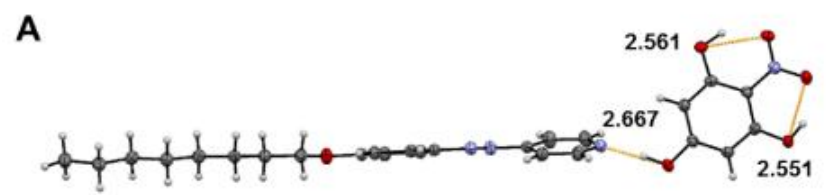

B

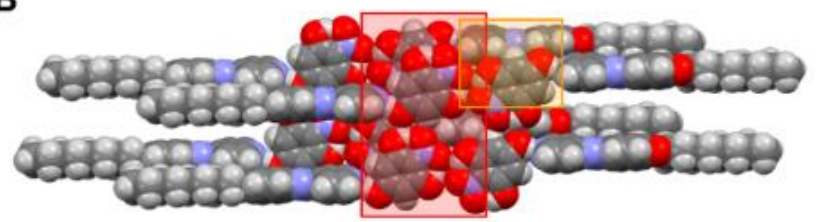

Fig. 6. Representative views of the molecular structure (A) and crystalline packing (B) of $\mathrm{NO}_{2}$-PHG $\cdots(\text { Ap-8) })_{1}$ showing $\pi-\pi$ interactions (red box) among the core units and $\mathrm{CH}-\pi$ interactions between pyridyl hydrogens and $\mathbf{N O}_{2}$-PHG (orange box). The RAHB ring is highlighted with a green six-membered ring. Ellipsoids are displayed at $50 \%$ probability. Distances are given in $\AA$.

\section{Improving the Liquid Crystalline Properties by RAHB}

Based on the crystallographic findings we changed the ratio between $\boldsymbol{m}$ CO-PHG and Ap-n to $1: 2$ leading to 12 new LC materials. Comparing the thermal properties of the $1: 2$ complexes with the previously described 1:3 complexes reveals significantly extended temperature ranges for the smectic phases (up to $\Delta T_{\max }=48{ }^{\circ} \mathrm{C}$ for the $1: 2$ assemblies instead of $\Delta \mathrm{T}_{\max }=20^{\circ} \mathrm{C}$ for the $1: 3$ (Fig. 4). This result clearly shows the relevance of the $1: 2$ ratio between hydrogen bond donating and accepting units. For the 1:3 assemblies we assume an interference of the third azopyridine unit with the RAHB, which results in a disturbance of the formation of the intramolecular hydrogen bond. In contrast, the RAHB increases the rigidity of the mesogenic core and its conjugation. ${ }^{5 a}$ Further correlation with the length of the alkanoyl group at the core unit shows a lowering of the transition temperatures from smectic to crystalline phase, which we attribute to the extension of the aliphatic chain.

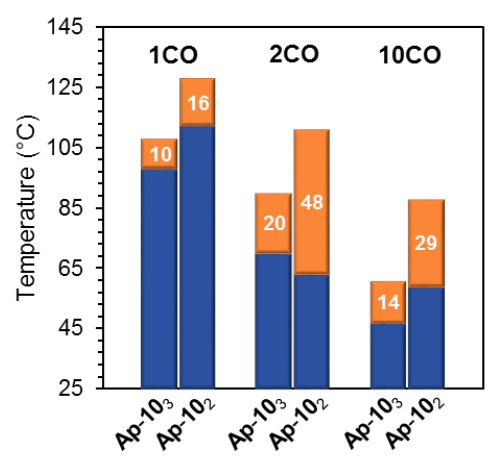

Fig. 4. Correlation of the transition temperatures and temperature ranges of the smectic phases with the length of the alkyl group at the $\mathbf{m C O}$-PHG core and the stoichiometry between core and side chain units. The upper limit for the mesophase range represent the clearing point of the assembly.

In analogy to the findings made for the carbonyl-functionalized assemblies, the stoichiometry of the $\mathbf{N O}_{2}$-PHG complexes was adjusted to a 1:1 ratio and the obtained assemblies were analysed with respect to their mesomorphic behaviour. Also in this case a mesophase was observed, however, in a very narrow temperature range $\left(\Delta \mathrm{T}_{X}=\sim 1^{\circ} \mathrm{C}\right)$, which was not observed for the 1:3 assemblies (Figure 1C). Although the RAHB has already been exploited by Meijer et al. to stabilize large discotic assemblies, ${ }^{23}$ herein, we used this concept for the first time to improve the liquid crystalline properties of supramolecular multicomponent mesogens. It is evident that intramolecular hydrogen bonding plays an essential role in the stabilization of the liquid crystalline phase by RAHB.

Being aware of this effect we designed a PHG-based core moiety, which is able to form intramolecular hydrogen bonds extending the rigidity and conjugation of the core, while still being capable to assemble with three azopyridine side chains. Therefore, the $\mathbf{N O}_{\mathrm{H}^{-}}$PHG was synthesized and co-crystallized with Ap-6. The crystal structure of $\mathbf{N O}_{\mathrm{H}^{-}}$PHG $\cdots(\mathbf{A p}-\mathbf{6})_{3}$ proves the $1: 3$ ratio of the hydrogen-bonded assembly $\left(\mathrm{OH} \cdots \mathrm{N}_{\text {pyr }}=2.65-2.78 \AA\right.$, Fig. 7). One of the phenolic $\mathrm{OH}$ groups forms an intramolecular hydrogen bond to the oxime nitrogen $\left(\mathrm{OH} \cdots \mathrm{N}_{\text {pyr }}=2.642 \AA\right)$ yielding a six-membered ring.

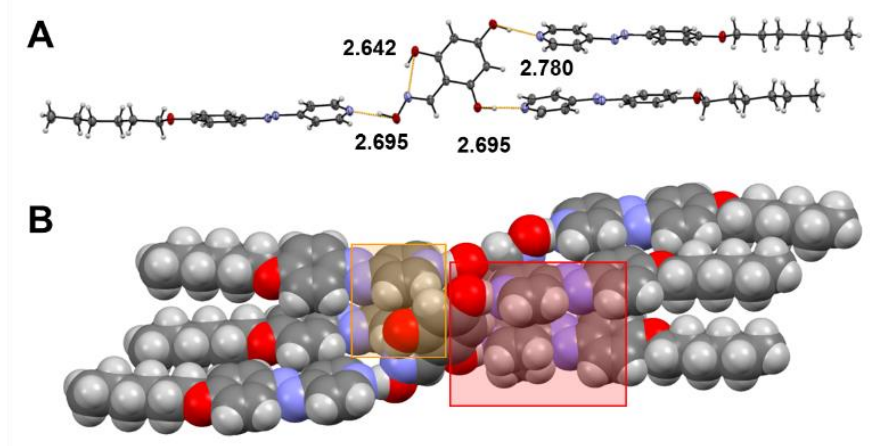

Fig. 7. Representative views of the molecular structure (A) and crystalline packing (B) of $\mathbf{N O}_{\mathrm{H}}$-PHG $\cdots(\mathbf{A p}-6)_{3}$ showing $\pi-\pi$ interactions (red and orange box) among the core units and $\mathrm{CH}-\pi$ forces between pyridyl hydrogens and $\mathrm{NO}_{2}$-PHG (orange box). The RAHB ring is highlighted with a green six-membered ring. Ellipsoids are displayed at $50 \%$ probability. Distances are given in $\AA$ (Colour chart: $\mathrm{C}=$ grey, $\mathrm{H}=$ white, $\mathrm{O}=$ red, $\mathrm{N}=$ purple). 
Interestingly, the $\mathbf{N O}_{\mathrm{H}^{-}}$PHG based assemblies revealed a broad temperature range for the nematic phase $\left(\Delta T_{\max }=53^{\circ} \mathrm{C}\right)$, which we attribute to the extension of the core unit by RAHB, which may in-turn increase the dipole moment of the core moiety by intramolecular hydrogen bonding.

\section{SAXS study}

In order to investigate the obtained hydrogen-bonded liquid crystals comprehensively $2 D$-X-ray scattering analysis was performed for representative examples (see ESI, chapter 6.15). The series of the halogenated $\mathbf{Y}$-PHG $\cdots(\mathbf{A p}-\mathbf{8})_{3}$ assemblies $(Y=$ $\mathrm{F}, \mathrm{Cl}, \mathrm{Br}$ ) showed two diffuse halos in the small-angle region $\left(2 \theta=2.8^{\circ}\right)$, which prove the nematic character of the mesophase. Compared to the non-functionalized PHG $\cdots(\mathbf{A p - 8})_{3}$ (Fig. 8, c) the $d$-spacing is slightly increased $(3.88 \mathrm{~nm})$, which can be explained by stronger stretching of the $\lambda$-folded conformation. ${ }^{24}$ No differences in the real space distances were found for the $\boldsymbol{F}$ - and the $\mathbf{B r}$-PHG-based assemblies indicating analogous mesogenic structures in the nematic phase (Fig. 8A, B). Furthermore, it suggests that the differences in the liquid crystalline behaviour are exclusively a result of the electronic anisotropy. However, radially averaged scattering plots reveal a sharpening of the diffuse peak in the small angle region, which suggests an increase of the molecular order of the bromine-based mesogens (Fig. 8B).

In contrast to the assemblies of the halogenated PHG units, the $\mathbf{C N}$-PHG $\cdots(\mathbf{A p}-\mathbf{1 0})_{3}$ assemblies show a sharp diffraction ring at $1.8^{\circ}$ supporting the present of a smectic phase (see ESI). This corresponds to a $d$ spacing of $4.82 \mathrm{~nm}$, which correlates with the layer distance in the smectic phase. Assuming the $\lambda$ folded conformation for these assemblies ${ }^{24}$ gives a length of $5.44 \mathrm{~nm}$ with Ap-10 (Supporting Figure S1). We suggest a monolayered arrangement of the assemblies with interdigitated side chains in the smectic phase (Fig. 9, left model). ${ }^{25,9 d}$
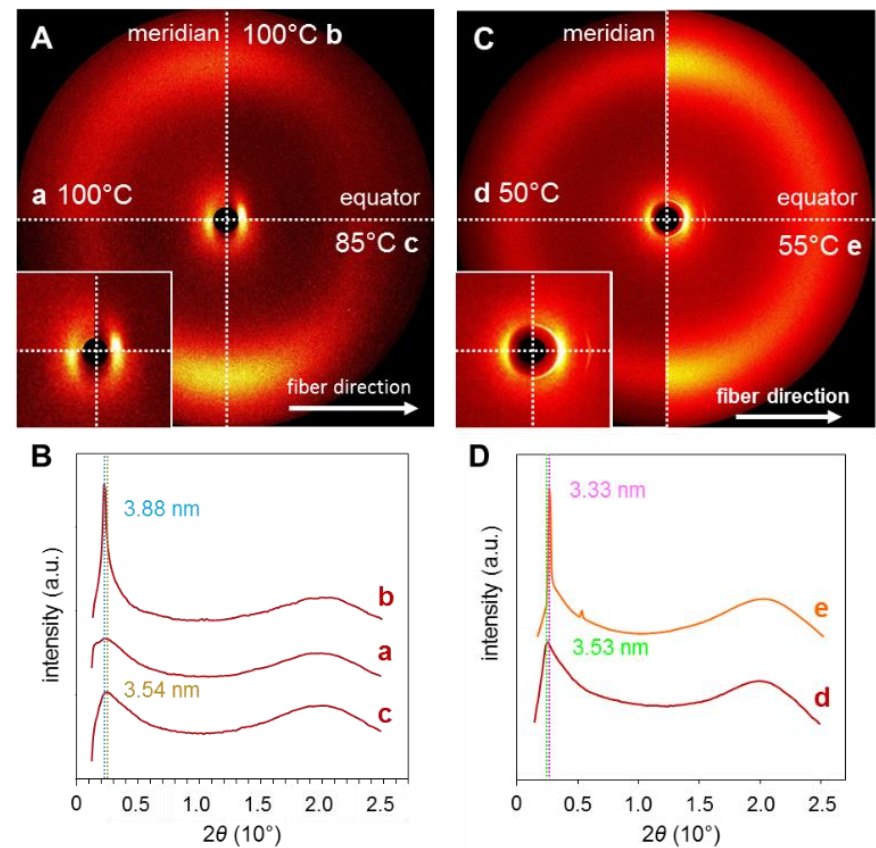

Fig. 8. Cut-out of $2 D$-Xray scattering patterns $(A, C)$ at corresponding temperature as well as radially averaged scattering plots (B, D) of $\boldsymbol{Y}$-PHG $\cdots(\mathbf{A p}-\mathbf{8})_{3}$ with $\mathrm{Y}=\mathrm{F}(\mathrm{a}), \mathrm{Br}(\mathrm{b})$, $H(c), 10 C(d)$ and $10 C O$ (e) reveal the structural morphology of the mesophases.

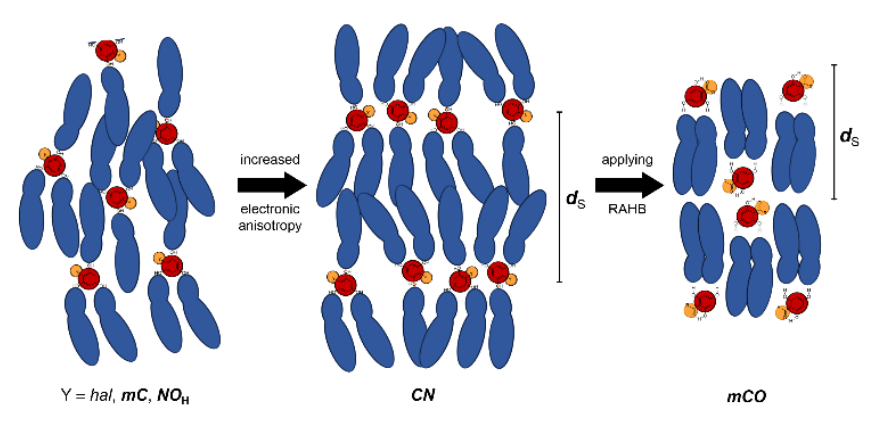

Fig. 9. Proposed models for the mesomorphic order of $\boldsymbol{Y}$-PHG-assemblies in the monolayered (left) and bi-layered (right) smectic as well as nematic phases (centre), controlled by the introduced group at the core. Hydrogen-accepting groups next to hydrogen bonding groups are capable to form resonance-assisted hydrogen bonds blocking the supramolecular binding site.

Studying the 1C-PHG and 10C-PHG assemblies yields virtually the same $d$-spacings $\left(d_{S}(\mathrm{~nm}), 1 C\right.$-PHG vs. 10C-PHG: 3.69 and 3.53 ) in the nematic phase (Fig. 8C, D). This implies thus that the extra alkyl group had no significant influence on the orientation of the mesogens in the nematic phase. Similar finding were also found for the alkanoyl-based assemblies. The real space distances obtained for the assemblies with $\mathbf{2 C O}$ - and 10CO-PHG cores and three Ap-8 units correspond to $3.33 \mathrm{~nm}$ (Fig. 8, e). The much shorter $d_{\mathrm{s}}$-values give an indirect prove for the formation of a different supramolecular mesogen with two azopyridine arms as supported by the corresponding crystal structure of 2CO-PHG $\cdots(\mathbf{A p}-3)_{2}$. Correlating the molecular length estimated for a $\mathbf{m C O}$-PHG $\cdots(\mathbf{A p}-\mathbf{8})_{2}$ assembly $(L=2.79 \mathrm{~nm}$, Supporting Fig. S1) with the $d$-spacing gives an $d_{\mathrm{S}} / L$ ratio of 1.2 , which suggests monolayers (Fig. 9, right model). ${ }^{25}$

\section{Conclusions}

In conclusion, we report a detailed structure-property relationship study on the impact of the substituent at the core moiety on the liquid crystalline behaviour of supramolecular Hekates formed by hydrogen bonding. In analogy to conventional liquid crystals, the dipole moment of the hydrogen bond donating core moiety needs to be high to yield assemblies with mesophases over a broad temperature ranges. However, more interesting are the findings on the role of intramolecular hydrogen bonding. Carbonyl, nitro and oxime groups next to the hydrogen donating groups are capable forming resonance-assisted hydrogen bonds, which counteracts the self-assembly behaviour of the 1:3 systems. However, adapting the number of hydrogen bond accepting side chains to the number of accessible hydrogen bond donating groups yields materials with improved liquid crystalline properties. Crystallographic results prove that the formation of the RAHB causes an extension/rigidification of 
the mesogenic core (rigidity), which widens the temperature ranges of the mesophases. The concept of RAHB was used to design an oxime based core moiety ( $\left.\mathbf{N O}_{\mathrm{H}^{-}} \mathbf{P H G}\right)$, which yielded 1:3 assemblies with azopyridine with broad mesophases. We expect these outcomes to be highly important for future studies on hydrogen-bonded liquid crystals, given the importance of phenol-based tectons in supramolecular design.

\section{Conflicts of interest}

There are no conflicts to declare.

\section{Acknowledgements}

We thank the Professor-Werdelmann foundation and the Fonds der Chemischen Industrie for generous financial support.

\section{Notes and references}

1 Special Issue Supramolecular Chemistry and SelfAssembly, 2002, http://science.sciencemag.org/content/295/5564, (accessed March 2019).

2(a) X. Yan, F. Wang, B. Zheng and F. Huang, Chem. Soc. Rev., 2012, 41, 6042-6065; (b) Y. Yang and M. W. Urban, Chem. Soc. Rev., 2013, 42, 7446-7467.

3(a) G. A. Jeffrey and W. Saenger, Hydrogen bonding in biological structures, Springer Science \& Business Media, 1991; (b) G. R. Desiraju and T. Steiner, The weak hydrogen bond: in structural chemistry and biology, International Union of Crystallography, 1999.

4(a) C. M. Paleos and D. Tsiourvas, Liq. Cryst., 2001, 28, 11271161; (b) W. He, G. Pan, Z. Yang, D. Zhao, G. Niu, W. Huang, X. Yuan, J. Guo, H. Cao and H. Yang, Adv. Mater., 2009, 21, 2050-2053.

5(a) T. Kato and J. M. Frechet, J. Am. Chem. Soc., 1989, 111, 8533-8534; (b) U. Kumar, T. Kato and J. M. J. Frechet, J. Am. Chem. Soc., 1992, 114, 6630-6639.

6(a) D. W. Bruce, in Supramol. Chem., John Wiley \& Sons, 2012, DOI: doi:10.1002/9780470661345.smc149; (b) J. P. W. Wong, A. C. Whitwood and D. W. Bruce, Chem. Eur. J., 2012, 18, 16073-16089.

7(a) H.-J. Sun, S. Zhang and V. Percec, Chem. Soc. Rev., 2015, 44, 3900-3923; (b) B. M. Rosen, C. J. Wilson, D. A. Wilson, M. Peterca, M. R. Imam and V. Percec, Chem. Rev., 2009, 109, 6275-6540; (c) V. Percec, A. E. Dulcey, V. S. K. Balagurusamy, Y. Miura, J. Smidrkal, M. Peterca, S. Nummelin, U. Edlund, S. D. Hudson, P. A. Heiney, H. Duan, S. N. Magonov and S. A. Vinogradov, Nature, 2004, 430, 764.

8(a) M. Lehmann, in Liquid Crystals: Materials Design and Selfassembly, ed. C. Tschierske, Springer Berlin Heidelberg, Berlin, Heidelberg, 2012, DOI: 10.1007/128_2011_266, pp. 193-223; (b) M. Lehmann, P. Maier, M. Grüne and M. Hügel, Chem. Eur. J., 2017, 23, 1060-1068; (c) P. Maier,
M. Grüne and M. Lehmann, Chem. Eur. J., 2017, 23, 10181022.

9(a) M. Pfletscher, C. Wolper, J. S. Gutmann, M. Mezger and M. Giese, Chem. Commun., 2016, 52, 8549-8552; (b)M. Spengler, R. Y. Dong, C. A. Michal, M. Pfletscher and M. Giese, J. Mater. Chem. C, 2017, 5, 2235-2239; (c) M. Pfletscher, S. Hölscher, C. Wölper, M. Mezger and M. Giese, Chem. Mater., 2017, 29, 8462-8471; (d) M. Pfletscher, M. Mezger and M. Giese, Soft Matter, 2018, 14,6214-6221; (e) M. Saccone, M. Pfletscher, E. Dautzenberg, R. Y. Dong, C. A. Michal and M. Giese, J.

Mater. Chem. C, 2019, 7, 3150-3153. and C. V. Yelamaggad, J. Org. Chem., 2013, 78, 527-544.

K. T. Mahmudov and A. J. L. Pombeiro, Chem. Eur. J., 2016, 22, 16356-16398.

A. L. Hickey and C. N. Rowley, J. Phys. Chem. A, 2014, 118, 3678-3687.

13 R. F. W. Bader, M. T. Carroll, J. R. Cheeseman and C. Chang, J. Am. Chem. Soc., 1987, 109, 7968-7979.

14 J. S. Murray and P. Politzer, Croat. Chem. Acta, 2009, 82, 267-275.

15 M. Bremer, P. Kirsch, M. Klasen-Memmer and K. Tarumi, Angew. Chem. Int. Ed., 2013, 52, 8880-8896.

16 M. L. Tang and Z. Bao, Chem. Mater., 2011, 23, 446-455.

17 S. Purser, P. R. Moore, S. Swallow and V. Gouverneur, Chem. Soc. Rev., 2008, 37, 320-330.

18 S. Alvarez, Dalton Trans., 2013, 42, 8617-8636.

19 M. Saccone, K. Kuntze, Z. Ahmed, A. Siiskonen, M. Giese and A. Priimagi, J. Mater. Chem. C, 2018, 6, 9958-9963.

20 D. T. Clark, J. N. Murrell and J. M. Tedder, J. Chem. Soc., 1963, DOI: 10.1039/JR9630001250, 1250-1253.

21(a) S. Z. Mohammady, R. I. Nessim, O. R. Shehab and M. M. Naoum, Liq. Cryst., 2005, 32, 477-482; (b) R. I. Nessim, M. M. Naoum, S. Z. Mohamed and M. I. Nessim, Liq. Cryst., 2004, 31, 649-654; (c) R. I. Nessim, Thermochim. Acta, 2002, 389, 49-58; (d) M. Roushdy, Liq. Cryst., 2004, 31, 371-375; (e) M. M. Naoum, T. Y. Labeeb, E. Happ and H. Seliger, Thermochim. Acta, 2002, 391, 151158; (f) M. M. Naoum, A. A. Fahmi, N. H. S. Ahmed and G. R. Saad, Liq. Cryst., 2015, 42, 1627-1637; (g) M. M. Naoum, A. A. Fahmi and M. A. Alaasar, Mol. Cryst. Liq. Cryst., 2009, 506, 22-33.

D. J. Price, K. Willis, T. Richardson, G. Ungar and D. W. Bruce, J. Mater. Chem., 1997, 7, 883-891.

23 A. R. A. Palmans, J. A. J. M. Vekemans, H. Fischer, R. A. Hikmet and E. W. Meijer, Chem. Eur. J., 1997, 3, 300-307.

24 M. Pfletscher, J. Wysoglad, J. S. Gutmann and M. Giese, RSC Adv., 2019, 9, 8444-8453.

25 J. W. Goodby, in Handbook of Liquid Crystals, 2014, DOI: doi:10.1002/9783527671403.hlc061. 